ACS Appl Mater Interfaces. 2019 June 19; 11(24): 21353-21359. doi:10.1021/acsami.9b04186.

\title{
Near-Infrared Fluorescent Endoscopic Image-Guided Photothermal Ablation Therapy of Colorectal Cancer Using Dual- Modal Gold Nanorods Targeting Tumor-Infiltrating Innate Immune Cells in a Transgenic TS4 CRE/APC'ox 468 Mouse Model
}

\author{
Elias Gournaris ${ }^{\dagger, \S, \|, ~ W o o r a m ~ P a r k}{ }^{\ddagger, \|}$, Soojeong Cho ${ }^{\ddagger}$, David J. Bentrem ${ }^{\dagger, \S}$, Andrew C. \\ Larson $^{\ddagger, \S}$, Dong-Hyun Kim ${ }^{\star}, \ddagger, \S$ \\ †Department of Surgery, Feinberg School of Medicine, Northwestern University, Chicago, Illinois \\ 60611, United States \\ FDepartment of Radiology, Feinberg School of Medicine, Northwestern University, Chicago, Illinois \\ 60611, United States \\ §Robert H. Lurie Comprehensive Cancer Center, Chicago, Illinois 60611, United States
}

\begin{abstract}
Colorectal cancer (CRC) is diagnosed with colonoscopy and treated with focal therapies. CRC is a good candidate for nanoparticle-mediated photothermal ablation (PTA) therapy. Herein, we developed a near-infrared fluorescent (NIRF) endoscopic image-guided PTA approach using a nanoparticle capable of simultaneously diagnosing and treating CRC. Dual-modal NIR heating and fluorescent gold nanorods (dual-modal GNRs) were synthesized by conjugation of GNRs to an NIRF probe. To validate the translational potential of our approach, a well-characterized transgenic TS4 CRE/ APC ${ }^{\text {lox } \triangle 468}$ colon cancer mouse model was used to carry out NIRF imageguided PTA using our dual-modal GNRs under clinically relevant conditions. Intravenously infused dual-modal GNRs were effectively targeted at colon polyps by immunogenic capturing of the GNRs within tumor-infiltrating innate immune cells. NIRF endoscopic image-guided PTA using the GNRs permitted successful detection and ablation of inflammatory colon polyps. NIRF endoscopy image-guided PTA using dual-modal GNRs can be utilized for diagnosis and treatment of CRC and various inflammatory diseases.
\end{abstract}

\section{Graphical Abstract}

\footnotetext{
"Corresponding Author: dhkim@northwestern.edu.

"E.G. and W.P. contributed equally to this work.

Supporting Information

The Supporting Information is available free of charge on the ACS Publications website at DOI: 10.1021/acsami.9b04186.

Digital and in vivo IR thermal camera images of a GNR-targeted polyp irradiated with an NIR laser (PDF)

The authors declare no competing financial interest.
} 


\section{NIRF endoscopic image-guided photothermal ablation}

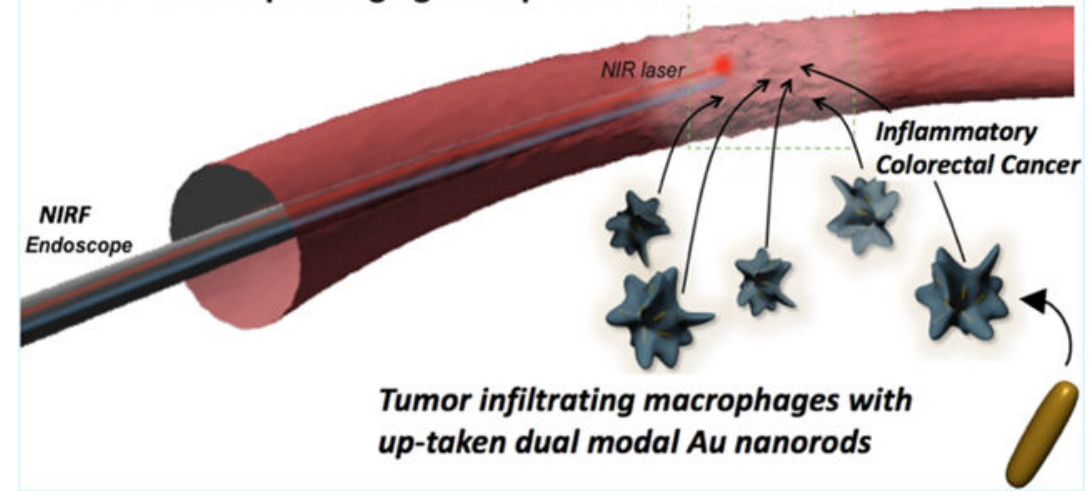

\section{Keywords}

colon cancer; endoscopic image-guided therapy; nanomedicine; interventional oncology; photothermal therapy

\section{INTRODUCTION}

Colorectal cancer (CRC) is the 3rd most common malignancy and 4th most common cause of cancer mortality worldwide. ${ }^{1} \mathrm{CRC}$ is also the $3 \mathrm{rd}$ most common cancer diagnosed in both men and women in the United States, despite important advances in detection, surgery, and chemotherapy. ${ }^{1,2}$ More than 1.4 million new cases of CRC are diagnosed worldwide each year. ${ }^{1}$ It was estimated to have caused roughly 50,630 deaths in $2018 .^{2}$ The most common treatment options for CRC are surgery, radiation therapy, and systemic chemo-therapy but the therapeutic efficacy of these approaches is limited and severe side effects are common.

Colonoscopy plays an important role in the localization of malignant lesions and subsequent identification at the time of surgery. It is widely accepted that early detection of a cancerous disease permits a broader spectrum of treatment options and improved outcomes. ${ }^{3-5}$ The use of high-definition white light endoscopy, the gold standard, has contributed to improved detection of early polypoid lesions. However, as white light detection of CRC is based upon structural changes, not upon functional criteria, it is still difficult to detect intraepithelial dysplasia and discriminate pseudo-polyps from polypoid dysplasia. Near-infrared fluorescent (NIRF) endoscopy with fluorescent probes may be a superior choice for the localization of CRC. Endoscopically detected pedunculated lesions are typically removed by using standard snare polypectomy, mechanical or electrocautery resection. ${ }^{6}$ As an alternative treatment, endoscopic laser therapy can provide relief of obstruction and bleeding in patients. ${ }^{7}$ Neodymium yttrium argon garnet (Nd:YAG) lasers $(1024 \mathrm{~nm})$ have been used to treat large, sessile, and benign polyps. ${ }^{7}$ However, nonspecific heating by the laser can result in coagulative necrosis of tissue with subsequent tissue sloughing and immediate vaporization of normal tissues. There are multiple potential complications during these laser ablation procedures including perirectal abscess formation, anal stenosis, and perforation. ${ }^{8,9}$ Perforations in the proximal colon result in spoilage of the free peritoneal space and often require surgical intervention. ${ }^{10}$ Recent studies showed no difference in life expectancy upon 
laser treatment of these lesions when compared to surgical resection. ${ }^{11,12}$ The greatest challenge for these therapeutic modalities is to treat only localized tumors while preserving adjacent tissues to minimize potential damage to normal tissues. ${ }^{13,14}$ Nanoparticle-mediated NIR photothermal ablation (PTA) therapy offers considerable promise for the detection and targeted treatment of inflammatory CRC, displaying robust pro-inflammatory innate immune cell infiltration and increased expression of proinflammatory cytokines. ${ }^{15-17}$ Recruited inflammatory macrophages, having an intrinsic disease-homing property, can be utilized to target nanoparticle delivery to CRC regions. ${ }^{18,19}$ Nanoparticles targeted at macrophages associated with proinflammatory substances have been utilized to visualize and treat various inflammatory diseases. ${ }^{20,21}$

Here, we report NIRF endoscopic PTA of CRC combined with immunogenic targeted NIRF gold nanorods (GNRs) for in situ identification and ablation of dysplastic lesions (Scheme 1). GNRs are considered a promising agent for photothermal therapy (PTT), given that these generate heat more rapidly compared to spherical gold nanoparticles, and GNRs can be readily synthesized with a tunable absorption wavelength. ${ }^{22}$ In our study, GNRs were tuned for efficient NIR photothermal heating and conjugated with NIR fluorophores (Cy 5.5) for CRC detection and PTA with a multichannel NIRF endoscope. To validate potential clinical translation, a clinically relevant transgenic TS4 CRE/APC ${ }^{\text {lox }} 468$ colon cancer mouse model was used to evaluate the efficacy of nanoparticle-mediated NIR endoscopic image-guided PTA. The selective accumulation of GNRs within inflammatory macrophages in CRC was characterized with in situ NIRF endoscopic imaging using cathepsin-activatable probes and immune fluorescenthistology analysis. Finally, NIRF endoscopic image-guided PTA using the GNRs was able to target dysplastic lesions in the transgenic colon cancer mouse model.

\section{MATERIALS AND METHODS}

\subsection{Materials.}

$\mathrm{HAuCl}_{4} \cdot 3 \mathrm{H}_{2} \mathrm{O}$ (99.9\%), L-ascorbic acid (AA; $\left.99 \%\right), \mathrm{NaBH}_{4}(99 \%), \mathrm{AgNO}_{3}(99 \%)$, and cetyltrimethylammonium bromide (CTAB; 99\%) were purchased from Sigma-Aldrich (St. Louis, MO, USA). Amine-PEG-thiol $\left(M_{\mathrm{w}} ; 5 \mathrm{kDa}\right)$ was obtained from Laysan Bio (Arab, Al, USA). Cyanine5.5 N-hydroxysuccinimide ester (Cy5.5-NHS) was purchased from Lumiprobe (FL, USA). ProSense 750 fluorescent activatable sensor technology was purchased from PerkinElmer (MA, USA).

\subsection{Preparation of Gold Nanoseeds.}

An aqueous $0.01 \mathrm{M}$ solution of $\mathrm{HAuCl}_{4} \cdot 3 \mathrm{H}_{2} \mathrm{O}(0.25 \mathrm{~mL})$ was added to a $0.10 \mathrm{M} \mathrm{CTAB}$ solution $(7.5 \mathrm{~mL})$. The solutions were carefully mixed through inversion. The color of the solution changed to bright brown-yellow. A cold $0.01 \mathrm{M} \mathrm{NaBH}_{4}$ solution $(0.06 \mathrm{~mL})$ was added to the solution and then mixed for $2 \mathrm{~min}$. The color of the mixture was immediately changed to a pale brown-yellow color and was further reacted for $2 \mathrm{~h}$ in a water bath maintained at $25^{\circ} \mathrm{C}$. 


\subsection{Seed-Mediated Preparation of GNR.}

CTAB $(0.10 \mathrm{M}, 4.75 \mathrm{~mL}), 0.01 \mathrm{M} \mathrm{HAuCl}_{4} \cdot 3 \mathrm{H}_{2} \mathrm{O}(0.20 \mathrm{~mL})$, and $0.01 \mathrm{M} \mathrm{AgNO}_{3}$ solution $(0.01-0.10 \mathrm{~mL})$ were added in a test tube sequentially and mixed gently. The color of the mixture changed to bright brown-yellow. Then, $0.10 \mathrm{M} \mathrm{AA}(0.032 \mathrm{~mL})$ was added to the mixture. Finally, after adding the seed solution $(0.01-0.05 \mathrm{~mL})$, the mixture was gently mixed for $10 \mathrm{~s}$, and the solution was left at room temperature for $3 \mathrm{~h}$. To synthesize surfacemodified GNRs with polyethylene glycols (PEGs), $2 \mathrm{mM}$ amine-PEG-thiol solution (100 $\mu \mathrm{L}$ ) was added to the $0.02 \mathrm{M}$ GNR solution $(10 \mathrm{~mL})$. The mixture was stirred for $3 \mathrm{~h}$ at room temperature. The PEG-modified GNRs were centrifuged for $10 \mathrm{~min}$ at $8500 \mathrm{rpm}$. After removing the supernatant, the PEG-modified GNRs were re-dispersed in PBS solution (5 $\mathrm{mL})$.

\subsection{NIR Fluorophore Labeling on the GNR.}

The PEG-modified GNRs ( $10 \mathrm{mg}$ ) were re-dispersed in $0.1 \mathrm{M}$ sodium bicarbonate buffer (1 $\mathrm{mL}, \mathrm{pH} 8.3)$, and Cy5.5-NHS (0.03 mg) was added. The mixture was left to react for $12 \mathrm{~h}$ at room temperature. To remove unconjugated Cy 5.5, dialysis was performed at $10 \mathrm{mM}$ PBS for $24 \mathrm{~h}$.

\subsection{Characterization of the Dual-Modal GNR.}

The absorption characteristics of the GNR were measured using a UV-vis spectrophotometer (cytation 3, BioTek, USA). The form of synthesized GNR was observed through transmission electron microscopy (TEM, Hitachi H-8000, Hitachi, Tokyo, Japan). To confirm the fluorescence of NIR fluorophore-labeled GNRs, samples were imaged with a fluorescent OV100 imaging system (Olympus, Tokyo, Japan). The fluorescent image was processed and analyzed using Image-Pro Plus Software (Olympus, Tokyo, Japan).

\subsection{Depth-Dependent Photothermal Heating.}

NIR diode-laser ( $808 \mathrm{~nm}, \mathrm{BWF5}, \mathrm{B} \& \mathrm{~W}$ Tek, Inc., DE, USA) connected to fiber optics was used to confirm the photothermal characteristics of the synthesized GNR. The GNR was redispersed to $1 \%$ agar phantom and then transferred to a plastic cuvette (Malvern, Herrenberg, Germany). Each sample was characterized under different-strength laser conditions. The thermal properties of GNR by NIR laser irradiation were investigated through an IR camera (ICI7320P, Beaumont, TX, USA). In this experiment, $808 \mathrm{~nm}$ laser at $1 \mathrm{~W} / \mathrm{cm}^{2}$ was used, and irradiation was performed at a distance of $1 \mathrm{~cm}$ from the sample. The laser was irradiated for $60 \mathrm{~s}$, and the laser power was adjusted by the distance between the optical fiber and the sample.

\subsection{In Vivo Real-Time Endoscopic Observation of Targeted Dual-Modal GNR on Polyps.}

A transgenic TS4 CRE/APC ${ }^{10 x \Delta 468}$ colon cancer mouse model was implemented for in vivo studies, typically producing 5-6 colon polyps at the age of 5-6 months. ${ }^{23,24}$ All procedures involving experimental animals were performed by protocols approved by the Northwestern University Institutional Animal Care and Use Committee. A cathepsin active probe $(2 \mathrm{nmol}$, ProSense 750) was injected into the mouse 16-24 h before endoscopy. For smooth endoscope insertion, PBS was injected before the insertion of the endoscope in the mouse 
colon. A fiberscope (BFXP60, Olympus, Tokyo, Japan) was used in this experiment. This dual laser fluorescence endoscope produces two distinctive excitation lights (680 and 750 $\mathrm{nm})$ with a little cross-talk between the channels. ${ }^{25}$ The images $(300 \times 300$ pixels $)$ obtained through endoscopy were analyzed and converted to video via NIH ImageJ software. The mean fluorescence intensity was calculated using the NIH ImageJ software after selecting three regions of interest $(30 \times 30$ pixels $)$ in the obtained images. The final calculated fluorescence intensity was plotted using GraphPad Prism5 software (GraphPad Software Inc, San Diego, CA, USA). ${ }^{26}$

\subsection{NIRF Endoscopy Imaging and Selective Dual-Modal GNR-Mediated PTA.}

Previously reported NIRF endoscopy was used for this study. ${ }^{25}$ Two laser light sources (680 and $750 \mathrm{~nm}$ ) with filters to avoid the cross-talk were fitted to the Olympus BF-XP60 fiberscope. The fiber endoscope has a $90^{\circ}$ field of view with a $2-50 \mathrm{~mm}$ local depth. The total length of the endoscope was $600 \mathrm{~mm}$, and its outer diameter was $2.8 \mathrm{~mm}$. The endoscope was equipped with a $1.2 \mathrm{~mm}$ instrument channel and was able to bend the end tip $180^{\circ}$ upwards and $130^{\circ}$ downwards. The fluorescent and reflectance light signals were processed by a high-sensitivity CCD camera through a splitter. The emitted fluorescent and the transmitted signal was recorded at 10 frames per second. ${ }^{16,27}$ The efficacy of NIRF endoscopy-guided PTT with dual-modal GNRs for the ablation of polyps was evaluated in TS4 CREI APC ${ }^{10 x} \triangle 468$ mouse models. Dual-modal GNRs $(\mathrm{Ex}=680 \mathrm{~nm})$ and a ProSense 750 $(\mathrm{Ex}=750 \mathrm{~nm})$ cathepsin activity probe for polyp detection were injected by the retro-orbital route. After $24 \mathrm{~h}$, the mice were subjected to endoscopy with a $680 \mathrm{~nm}$ laser to detect the focal distribution of dual-modal GNRs. Polyps were detected using a $750 \mathrm{~nm}$ laser. Images were collected and evaluated for focal increases of the emissions of the probes. Then, the NIR laser (power, $1 \mathrm{~W} / \mathrm{cm}^{2}$ for $10 \mathrm{~s}$ ) was irradiated to the polyps. The fine structure of the lesions before and after the PTT, and the focal distribution of dual-modal GNRs were verified with intravital microscopy and reflectance fluorescence.

\subsection{Histopathology Assessments.}

Colon polyps with mouse intestine were fixed in $10 \%$ formalin solution for $24 \mathrm{~h}$. The tissue specimens were sliced at $1 \mathrm{~mm}$ intervals and then sectioned to a thickness of $5 \mu \mathrm{m}$. H\&E and TUNEL staining were used to determine necrotic/apoptotic tumor tissues. The tissue was analyzed through a TissueFAXS microscope (TissueGnostics GmbH, Vienna, Austria). For immunofluorescence studies, sequential $5 \mu \mathrm{m}$ thick optimal cutting temperature (OCT) frozen tissue sections were stained with anti -F4/80 and Gr1 antibodies and counterstained with DAPI. The images were collected with the TissueFAXS microscope.

\section{RESULTS AND DISCUSSION}

\subsection{Synthesis of Dual-Modal GNRs.}

GNRs were synthesized through a two-step synthesis process. After synthesizing GNR capable of absorbing light in the NIR region, fluorescent molecules were conjugated to the surface of the GNR. First, the amount of $\mathrm{AgNO}_{3}$ in the synthesis process was adjusted to synthesize the GNR of appropriate size to absorb NIR wavelength light. As the amount of $\mathrm{AgNO}_{3}$ increased, the aspect ratio of the GNR also increased, and the absorption wavelength 
shifted to the NIR wavelength (Figure 1a). In this study, the GNR with the size of $12.7 \pm 3.4$ $\mathrm{nm}$ (width) and $47 \pm 9.3 \mathrm{~nm}$ (length) having the highest absorption at an NIR wavelength of around $800 \mathrm{~nm}$ was selected and used for further in vitro and in vivo experiments (Figure 1b). The aspect ratio of the GNR was 3.7. In order to introduce a fluorescent probe to the surface of GNRs for optical imaging and reduce their toxicity, cytotoxic surface ligands (i.e., $\mathrm{CTAB}$ ) of the GNR were replaced with biocompatible polyethyleneglycol (PEG, $5 \mathrm{kDa}$ ) molecules bi-functionalized with thiol and amine groups. The terminal amine group of PEG can improve the uptake by macrophages in vivo by making GNR surfaces positively charged. ${ }^{28,29}$ After the surface modification with PEG, it was determined that the GNRs contained 20000 polymers per particle according to the N-succinimidyl 3-(2-

pyridyldithio)propionate (SPDP) assay. ${ }^{30}$ An NIR probe (Cy 5.5; Cyto 680) was conjugated to the amine group at the end of the PEG molecule (Figure 1c). When examined with an in vivo imaging station (Olympus Corp., Tokyo, Japan), the Cy5.5-labeled GNRs showed an excellent fluorescent signal as anticipated (Figure 1d). This result implies that the Cy5.5GNRs are suitable for optical fluorescence imaging using NIRF endoscopy. Henceforth, in this report, the GNRs conjugated with NIR fluorescent probes will be described as dualmodal GNRs.

\subsection{Evaluation of the Photothermal Effects of Dual-Modal GNRs.}

To confirm the photothermal effects of the as-synthesized dual-modal GNRs, the nanoparticles were embedded in an agarose gel and irradiated with an NIR laser to evaluate temperature changes (Figure 1e). The tissue penetration rate of the laser source can greatly affect the ablation efficacy during photothermal therapy. ${ }^{31}$ The NIR wavelength $(808 \mathrm{~nm})$ laser used in this study is known to have high tissue permeability. ${ }^{32,33}$ A nanoparticleembedded agarose gel with a thickness of $1 \mathrm{~cm}$ was prepared and irradiated with an NIR laser at a distance of $1 \mathrm{~cm}$ from the agarose gel. Temperature changes of the gels were measured at different positions over time using a thermal camera while irradiating with the laser (Figure 1f). The temperature increase rate at $1 \mathrm{~cm}$ distance from the laser source demonstrated the fastest heating. All sites within $2 \mathrm{~cm}$ of where the dual-modal GNRs were embedded showed a tendency to increase with time when irradiated with the laser (Figure $1 \mathrm{~g}$ ). These results provided information about NIR laser power and distance-dependent spatial heating profiles between the laser source and lesions for effective PTA of epithelial and intraepithelial dysplasia.

\subsection{NIRF Endoscopic Detection of Polyps Using Dual-Modal GNRs.}

To perform an endoscopic image-guided PTA with dual modal GNRs in a colon cancer in vivo, a microendoscope device with a laser permitting dual excitation wavelengths was set up as described in refs. ${ }^{16,27}$ The tool channel of the BF-XP60 fiberscope was used to insert the fiber optic $808 \mathrm{~nm}$ NIR laser for photothermal therapy (Figure 2b). The tip of the fiberscope was guided to allow the laser to be placed on the tumor area. In this study, we used the transgenic TS4 CRE/APC ${ }^{10 x \Delta 68}$ mouse model. ${ }^{23}$ This transgenic mouse model faithfully develops only colon polyps. No polyps are detected in the small bowel, and the mice can live longer as they are not anemic as compared to adenomatous polyposis coli (APC) mice. To image colon polyps with NIRF endoscopy, an NIRF ProSense 750 probe, which is specifically activated by cathepsin enzymes in the tumor environment, was injected 
into the animal model. As shown in Figure $2 \mathrm{~b}$ (left), the tumor was imaged using the ProSense 750 probe. Then, the Cy5.5-labeled dual-modal GNRs were injected into the colon cancer mouse model. The accumulation of the injected dual-modal GNRs at the tumor site was confirmed by the co-localized fluorescence observed when compared to ProSense 750 NIRF endoscopy [Figure 2b (right)]. Macrophages, uptaking nanoparticles with innate phagocytotic properties, can serve as a "Trojan Horse" delivery vector of nano-medicine. 20,34 Macrophages capturing nanoparticles have strong potential for cancer therapy because the macrophages can by-pass nearly impermeable tissue barriers to reach many areas in the body where common nanoparticles cannot reach. ${ }^{34-37}$ The recognition of these rod-shaped gold nanoparticles by the macrophage system is consistent with previous reports. ${ }^{34,38,39}$ To confirm the recognition of the dual-modal GNRs by macrophages and accumulation in a tumor site, the polyp tissue was extracted for immunofluorescent study after GNR injection. Green fluorescence for the dual-modal GNPs and red fluorescence for macrophages overlapped in the tumor tissue (Figure 2c). This result indicates that the dual-modal GNRcaptured macrophages migrate to inflammatory colon cancer regions. Then, to examine the photothermal effect of dual-modal GNRs at the disease site, the GNRs were injected into the mice model via iv, and the small intestines with colon polyps from the mice were extracted to measure NIR photothermal heating. As shown in Figure 2d, the temperature of the tumor tissue was significantly increased compared to the surrounding tissue. GNR-mediated photothermal heating was also confirmed in vivo heating of the colon polyps during blood circulation (Supporting Information, Figure S1). These results indicate that the injected dualmodal GNRs are effectively targeted at tumors through tumor-infiltrating macrophages, thereby enabling tumor-specific PTA with NIRF endoscopic image guidance.

\subsection{In Vivo Therapeutic Efficacy of NIRF Endoscopic Image-Guided PTA Therapy with Dual-Modal GNRs in a Transgenic TS4 CRE/APClox $\triangle 468$ Colon Dysplasia Mouse Model.}

Finally, we confirmed the PTA therapeutic effect of NIRF endoscopic image-guided PTA therapy with dual-modal GNRs in a transgenic TS4 CRE/ APC lox 468 colon dysplasia mouse model. To induce a carcinogenic process in the colon, homologous recombination was performed to introduce loxP sites of the mouse APC ${ }^{l o x \Delta 468}$ gene. ${ }^{40,41}$ These transgenic mice are an appropriate animal model for studying inflammation and colonic polyposis.

After injecting the GNRs into mice, the colorectal polyps detected by Cy5.5-GNRs were selectively irradiated by an NIR laser through an NIRF endoscopic guidance with a ProSense 750 probe, as shown in the real-time NIR endoscopic images (Figure 3). As Step 1, a polyp was detected with Cy5.5 GNRs. The detected polyp was then readily irradiated with an NIR laser equipped within an endoscope. After a single session of laser irradiation, the polyp was successfully ablated (Figure 3). After the procedure, the entire colon of the animal model was extracted and histologically stained to confirm the treatment of colon polyps by the photothermal effect. The polyp area showed significant cell death according to H\&E and TUNEL staining (Figure 4a,b). The fluorescent immunohistochemistry of the treated tissue showed an interface area between normal tissues and the polyp, the latter colocalizing fluorescence from GNRs (red) and macrophages cells (green). The area with GNR-containing macrophage accumulation was selectively ablated after the NIR laser irradiation (Figure 4c). Neutrophil staining, one marker for inflammatory polyps, confirmed 
the preferential GNR distribution in the polyp area and precise ablation of the polyp. This result implies that the targeted dual-modal GNRs delivered via immunogenic macrophages can be used to effectively treat colon cancers via NIRF endoscopy-guided PTA with dualmodal GNRs.

\section{CONCLUSIONS}

Nanoparticle-mediated PTA has rapidly advanced from concept to clinical trials for the PTA treatment of various cancers. PTA combined with imaging tools to guide therapeutics and monitor the success of treatment will offer great potential for increasing therapeutic efficacy while decreasing off-target delivery to healthy tissue. In this study, NIRF endoscopic imageguided PTA was performed with NIR fluorophore-labeled GNRs for effective CRC treatment. The dual-modal GNRs, capable of simultaneously performing NIR fluorescent imaging and PTA, were designed by conjugation of rod-shaped gold nanoparticles and $\mathrm{Cy}$ 5.5 NIR fluorescent molecules. The synthesized dual-modal GNRs were not only capable of NIR fluorescent imaging but also exhibited effective photothermal effects upon irradiation using an endoscopic NIR wavelength laser. A translational transgenic mouse model successfully demonstrated that dual-modal GNRs could target inflammatory polyps. Finally, NIRF endoscopic image-guided PTA with the dual-modal GNRs achieved precise ablation of polyps. This NIRF endoscopic image guidance approach combined with tumor-targeted fluorescent GNRs have the potential for the detection and treatment of CRC and various inflammatory diseases. Various new imaging techniques such as MRI, CT, photoacoustic imaging, and multifunctional nano-agents capable of highly efficient photothermal heating and imaging will further develop this approach for the treatment of CRC.

\section{Supplementary Material}

Refer to Web version on PubMed Central for supplementary material.

\section{ACKNOWLEDGMENTS}

This work was supported by grants R01CA218659 and R01EB026207 from the National Cancer Institute and National Institute of Biomedical Imaging and Bioengineering.

\section{REFERENCES}

(1). Torre LA; Bray F; Siegel RL; Ferlay J; Lortet-Tieulent J; Jemal A Global Cancer Statistics, 2012. Ca-Cancer J. Clin 2015, 65, 87-108. [PubMed: 25651787]

(2). Kohler BA; Sherman RL; Howlader N; Jemal A; Ryerson AB; Henry KA; Boscoe FP; Cronin KA; Lake A; Noone A-M; Henley SJ; Eheman CR; Anderson RN; Penberthy L Annual Report to the Nation on the Status of Cancer, 1975-2011, Featuring Incidence of Breast Cancer Subtypes by Race/Ethnicity, Poverty, and State. J. Natl. Cancer Inst 2015, 107, dvj048.

(3). Rutter MD Surveillance Programmes for Neoplasia in Colitis.J. Gastroenterol 2011, 46, 1-5.

(4). Winawer SJ; Zauber AG; Ho MN; O’Brien MJ; Gottlieb LS; Sternberg SS; Waye JD; Schapiro M; Bond JH; Panish JF; Ackroyd F; Shike M; Kurtz RC; Hornsby-Lewis L; Gerdes H; Stewart ET Prevention of Colorectal-Cancer by Colonoscopic Polypectomy. N. Engl. J. Med 1993, 329, 1977-1981. [PubMed: 8247072]

(5). Zauber AG; Winawer SJ; O’Brien MJ; Lansdorp-Vogelaar I; van Ballegooijen M; Hankey BF; Shi WJ; Bond JH; Schapiro M; Panish JF; Steward ET; Waye JD Colonoscopic Polypectomy and 
Long-Term Prevention of Colorectal Cancer Deaths. N. Engl. J. Med 2012, 366, 687-696. [PubMed: 22356322]

(6). Rex D; Bond JH; Winawer S; Levin TR; Burt RW; Johnson DA; Kirk LM; Litlin S; Lieberman DA; Waye JD; Church J; Marshall JB; Riddell RH; Cancer, U.S. Multi-Society Task Force on Colorectal Cancer. Quality in the Technical Performance of Colonoscopy and the Continuous Quality Improvement Process for Colonoscopy: Recommendations of the U.S. Multi-Society Task Force on Colorectal Cancer. Am. J. Gastroenterol 2002, 97, 1296-1308. [PubMed: 12094842]

(7). Oxenberg J; Hochwald SN; Nurkin S Ablative Therapies for Colorectal Polyps and Malignancy. BioMed Res. Int 2014, 2014, 986352. [PubMed: 25089281]

(8). Low DE; Kozarek RA; Ball TJ; Patterson DJ; Hill LD Colorectal Neodymium-YAG Photoablative Therapy. Comparing Applications and Complications on Both Sides of the Peritoneal Reflection. Arch. Surg 1989, 124, 684-688. [PubMed: 2786403]

(9). Mathus-Vliegen EMH; Tytgat GNJ The Potential and Limitations of Laser Photoablation of Colorectal Adenomas. Gastrointest. Endosc. 1991, 37, 9-17.

(10). Pritikin J; Weinman D; Harmatz A; Young H Endoscopic Laser Therapy in Gastroenterology. West. J. Med 1992, 157, 48-54. [PubMed: 1413743]

(11). Brunetaud JM; Mosquet L; Houcke M; Scopelliti JA; Rance FA; Cortot A; Paris JC Villous Adenomas of the Rectum. Results of Endoscopic Treatment with Argon and Nd:YAG Lasers. Gastroenterology 1985, 89, 832-837. [PubMed: 4029563]

(12). Ronnekleiv-Kelly SM; Kennedy GD Management of Stage IV Rectal Cancer: Palliative Options. World J. Gastroenterol 2011, 17, 835-847. [PubMed: 21412493]

(13). Zhao K; Cho S; Procissi D; Larson AC; Kim D-H Noninvasive Monitoring of Branched Au Nanoparticle-mediated Photo-thermal Ablation. J. Biomed. Mater. Res. B Appl. Biomater 2017, 105, 2352-2359. [PubMed: 27520071]

(14). Kim D-H; Larson AC Deoxycholate Bile Acid Directed Synthesis of Branched Au Nanostructures for Near Infrared Photothermal Ablation. Biomaterials 2015, 56, 154-164. [PubMed: 25934288]

(15). Fujiwara N; Kobayashi K Macrophages in Inflammation. Curr. Drug Targets-Inflamm. Allergy 2005, 4, 281-286. [PubMed: 16101534]

(16). Gounaris E; Martin J; Ishihara Y; Khan MW; Lee G; Sinh P; Chen EZ; Angarone M; Weissleder R; Khazaie K; Barrett TA Fluorescence Endoscopy of Cathepsin Activity Discriminates Dysplasia from Colitis. Inflamm. Bowel Dis. 2013, 19, 1339-1345.

(17). White SB; Kim D-H; Guo Y; Li W; Yang Y; Chen J; Gogineni VR; Larson AC Biofunctionalized Hybrid Magnetic Gold Nanoparticles as Catalysts for Photothermal Ablation of Colorectal Liver Metastases. Radiology 2017, 285, 809-819. [PubMed: 28707960]

(18). Hasan DM; Mahaney KB; Magnotta VA; Kung DK; Lawton MT; Hashimoto T; Winn HR; Saloner D; Martin A; Gahramanov S; Dósa E; Neuwelt E; Young WL Macrophage Imaging Within Human Cerebral Aneurysms Wall Using Ferumoxytol-Enhanced MRI: A Pilot Study. Arterioscler. Thromb. Vasc. Biol 2012, 32, 1032-1038. [PubMed: 22328774]

(19). Fang J; Nakamura H; Maeda H The EPR Effect: Unique Features of Tumor Blood Vessels for Drug Delivery, Factors Involved, and Limitations and Augmentation of the Effect. Adv. Drug Deliv. Rev 2011, 63, 136-151. [PubMed: 20441782]

(20). Choi J; Kim H-Y; Ju EJ; Jung J; Park J; Chung H-K; Lee JS; Lee JS; Park HJ; Song SY; Jeong SY; Choi EK Use of Macrophages to Deliver Therapeutic and Imaging Contrast Agents to Tumors. Biomaterials 2012, 33, 4195-4203. [PubMed: 22398206]

(21). Choi M-R; Stanton-Maxey KJ; Stanley JK; Levin CS; Bardhan R; Akin D; Badve S; Sturgis J; Robinson JP; Bashir R; Halas NJ; Clare SE A Cellular Trojan Horse for Delivery of Therapeutic Nanoparticles into Tumors. Nano Lett. 2007, 7, 3759-3765. [PubMed: 17979310]

(22). Pissuwan D; Valenzuela SM; Killingsworth MC; Xu X; Cortie MB Targeted Destruction of Murine Macrophage Cells with Bioconjugated Gold Nanorods. J. Nanopart. Res 2007, 9, 11091124.

(23). Zeineldin M; Neufeld KL More than Two Decades of Apc Modeling in Rodents. Biochim. Biophys. Acta 2013, 1836, 80-89. [PubMed: 23333833] 
(24). Khazaie K; Zadeh M; Khan MW; Bere P; Gounari F; Dennis K; Blatner NR; Owen JL; Klaenhammer TR; Mohamadzadeh M Abating Colon Cancer Polyposis by Lactobacillus Acidophilus Deficient in Lipoteichoic Acid. Proc. Natl. Acad. Sci. U.S.A 2012, 109, 1046210467. [PubMed: 22689992]

(25). Shrivastav M; Gounaris E; Khan MW; Ko J; Ryu SH; Bogyo M; Larson A; Barret TA; Bentrem DJ Validation of Near Infrared Fluorescence (NIRF) Probes In Vivo with Dual Laser NIRF Endoscope. PLoS One 2018, 13, No. e0206568.

(26). Gounaris E; Tung CH; Restaino C; Maehr R; Kohler R; Joyce JA; Plough HL; Barrett TA; Weissleder R; Khazaie K Live imaging of cysteine-cathepsin activity reveals dynamics of focal inflammation, angiogenesis, and polyp growth. PLoS One 2008, 3, No. e2916.

(27). Gounaris E; Ishihara Y; Shrivastrav M; Bentrem D; Barrett TA Near-Infrared Fluorescence Endoscopy to Detect Dysplastic Lesions in the Mouse Colon. Methods Mol. Biol 2016, 1422, 137-147. [PubMed: 27246029]

(28). Xiao K; Li Y; Luo J; Lee JS; Xiao W; Gonik AM; Agarwal RG; Lam KS The Effect of Surface Charge on In Vivo Biodistribution of PEG-oligocholic Acid Based Micellar Nanoparticles. Biomaterials 2011, 32, 3435-3446. [PubMed: 21295849]

(29). Blanco E; Shen H; Ferrari M Principles of Nanoparticle Design for Overcoming Biological Barriers to Drug Delivery. Nat. Biotechnol 2015, 33, 941-951. [PubMed: 26348965]

(30). Fang C; Bhattarai N; Sun C; Zhang M Functionalized Nanoparticles with Long-term Stability in Biological Media. Small 2009, 5, 1637-1641. [PubMed: 19334014]

(31). Huang X; El-Sayed IH; Qian W; El-Sayed MA Cancer Cell Imaging and Photothermal Therapy in the Near-infrared Region by Using Gold Nanorods. J. Am. Chem. Soc 2006, 128, 2115-2120. [PubMed: 16464114]

(32). O'Neal DP; Hirsch LR; Halas NJ; Payne JD; West JL Photo-thermal Tumor Ablation in Mice Using Near Infrared-absorbing Nanoparticles. Cancer Lett. 2004, 209, 171-176. [PubMed: 15159019]

(33). Dickerson EB; Dreaden EC; Huang X; El-Sayed IH; Chu H; Pushpanketh S; McDonald JF; ElSayed MA Gold Nanorod Assisted Near-infrared Plasmonic Photothermal Therapy (PPTT) of Squamous Cell Carcinoma in Mice. Cancer Lett. 2008, 269, 57-66. [PubMed: 18541363]

(34). Li Z; Huang H; Tang S; Li Y; Yu X-F; Wang H; Li P; Sun Z; Zhang H; Liu C; Chu PK Small Gold Nanorods Laden Macrophages for Enhanced Tumor Coverage in Photothermal Therapy. Biomaterials 2016, 74, 144-154. [PubMed: 26454052]

(35). Choi M-R; Stanton-Maxey KJ; Stanley JK; Levin CS; Bardhan R; Akin D; Badve S; Sturgis J; Robinson JP; Bashir R Cellular Trojan Horse for Delivery of Therapeutic Nanoparticles into Tumors. Nano Lett. 2007, 7, 3759-3765. [PubMed: 17979310]

(36). Madsen SJ; Baek S-K; Makkouk AR; Krasieva T; Hirschberg H Macrophages as Cell-based Delivery Systems for Nanoshells in Photothermal Therapy. Ann. Biomed. Eng 2012, 40, 507515. [PubMed: 21979168]

(37). Mooney R; Roma L; Zhao D; Van Haute D; Garcia E; Kim SU; Annala AJ; Aboody KS; Berlin JM Neural Stem Cell-mediated Intratumoral Delivery of Gold Nanorods Improves Photothermal Therapy. ACS Nano 2014, 8, 12450-12460. [PubMed: 25375246]

(38). Bartneck M; Keul HA; Singh S; Czaja K; Bornemann J; Bockstaller M; Moeller M; ZwadloKlarwasser G; Groll J Rapid Uptake of Gold Nanorods by Primary Human Blood Phagocytes and Immunomodulatory Effects of Surface Chemistry. ACS Nano 2010, 4, 3073-3086. [PubMed: 20507158]

(39). Qin J; Peng Z; Li B; Ye K; Zhang Y; Yuan F; Yang X; Huang L; Hu J; Lu X Gold Nanorods as a Theranostic Platform for In Vitro and In Vivo Imaging and Photothermal Therapy of Inflammatory Macrophages. Nanoscale 2015, 7, 13991-14001. [PubMed: 26228112]

(40). Saam JR; Gordon JI Inducible Gene Knockouts in the Small Intestinal and Colonic Epithelium. J. Biol. Chem 1999, 274, 38071-38082. [PubMed: 10608876]

(41). Khazaie K; Zadeh M; Khan MW; Bere P; Gounari F; Dennis K; Blatner NR; Owen JL; Klaenhammer TR; Mohamadzadeh M Abating Colon Cancer Polyposis by Lactobacillus Acidophilus Deficient in Lipoteichoic Acid. Proc. Natl. Acad. Sci. U.S.A 2012, 109, 1046210467. [PubMed: 22689992] 
(a)

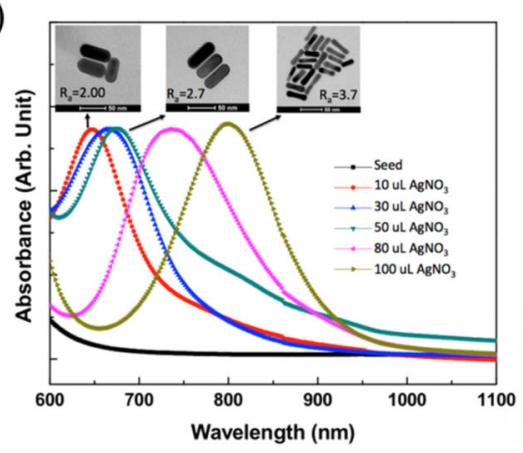

(e)

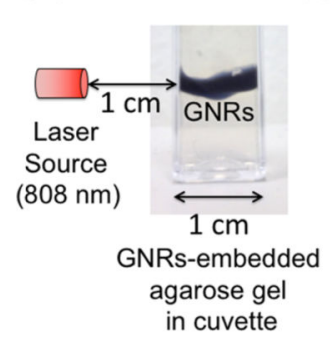

(f)

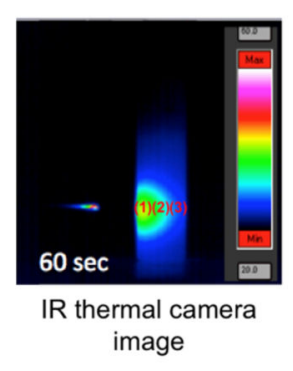

(b)

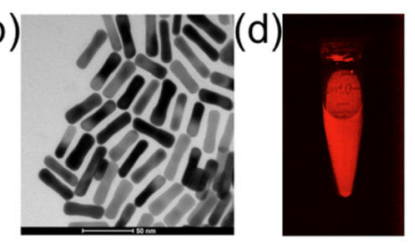

(C)

$\mathrm{SH}-\mathrm{NO}-\mathrm{NH}_{2}$ Thiol-PEG-Amine

Au nanorods

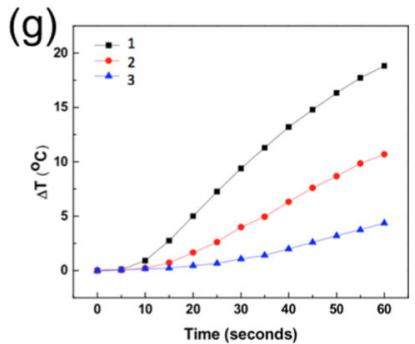

Figure 1.

Characterization of dual-modal GNRs. (a) UV-vis spectra of GNRs synthesized under different synthesis conditions (Inset: TEM images of synthesized GNRs). (b) TEM image of the PEG-coated GNRs. (c) Schematic illustration of dual-modal GNRs. After ligand exchange of the GNRs with PEG polymers bifunctionalized with thiol and amine groups, the NIR fluorescent probes are conjugated to the amine group at the end of the PEG. (d) Cy5.5 fluorescent image of the dual-modal GNRs. (e) Experimental setup for measurement of the photothermal effect by depth in artificial tissue (i.e., agarose gel). (f) Infrared thermal camera images of an agarose gel-containing dual-modal GNRs at $60 \mathrm{~s}$ after irradiation. (g) Temperatures recorded using infrared thermal camera images of the agarose gel at increasing distances from the laser irradiation site. (1-3) were measured using IR thermal camera images (Figure 1f) at a distance of 1, 1.5, and $2.0 \mathrm{~cm}$ from the laser source, respectively. 
(a)

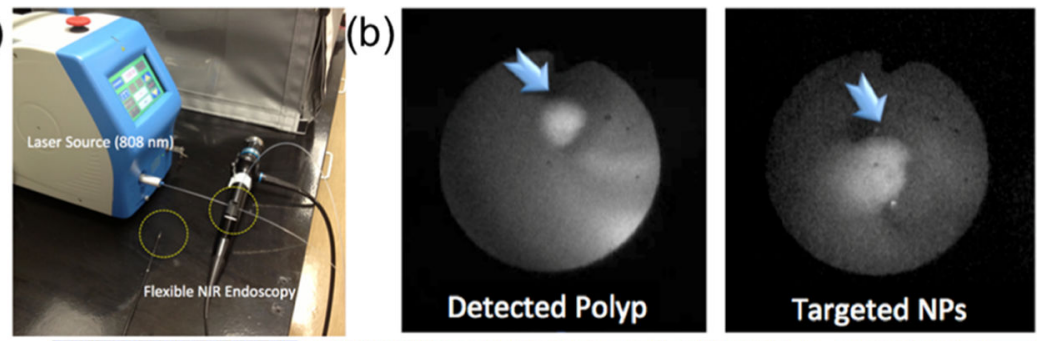

(C)

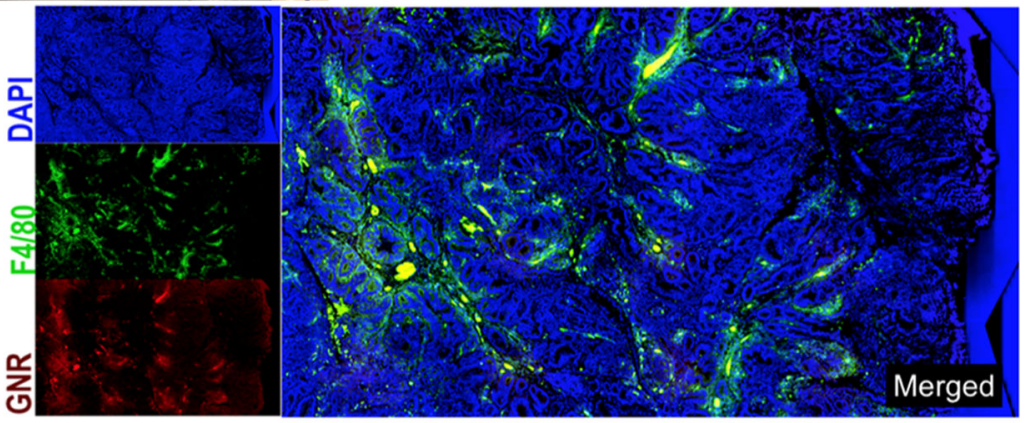

(d)
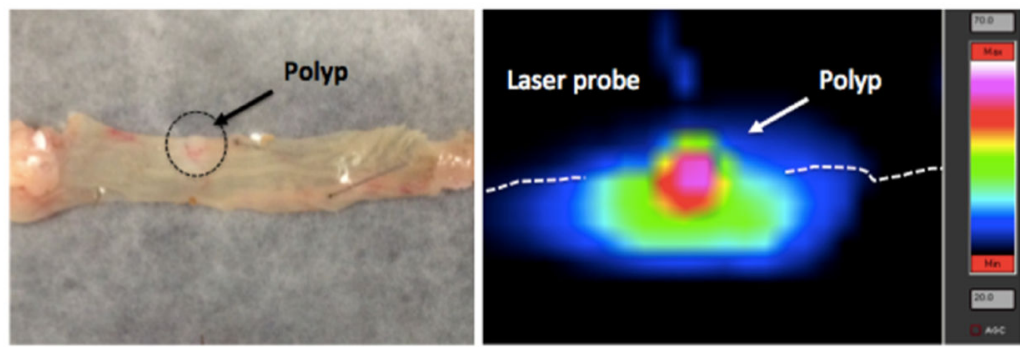

Figure 2.

NIR fluorescent endoscopic colon polyp detection with dual-modal GNRs. (a) Image of a fiber-type near-infrared laser ( $808 \mathrm{~nm}$ ) device connected to the endoscope. (b) In vivo NIRF endoscopic imaging of tumor tissue (i.e., colon polyp). (Left) The colon polyps were imaged using a ProSense 750 fluorescent probe that specifically detected cathepsin enzyme activity in cancer cells. (Right) The accumulated dual-modal GNRs labeled with Cy5.5 in the same colon polyps were imaged through the NIRF endoscope. (c) Fluorescent immunohistology images showing the distribution of dual-modal GNRs and macrophages in the colon polyps. Cy5.5 labeled with GNRs (green) and fluorescent F4/80 antibody (red), a marker for macrophages, are imaged with fluorescent microscopy, respectively (63× magnification). (d) In situ IR thermal camera image of a small intestine with colon polyps from a transgenic mouse colon cancer model. (Left) Digital photograph of a mouse intestine with colon polyps. (Right) IR thermal camera image when NIR laser is applied to the dual-modal GNRs upon targeted delivery to a colon polyp. 
Step 1. Real-time NIR Fluorescent Endoscopic Detection of Polyp with GNR (Cy5.5)

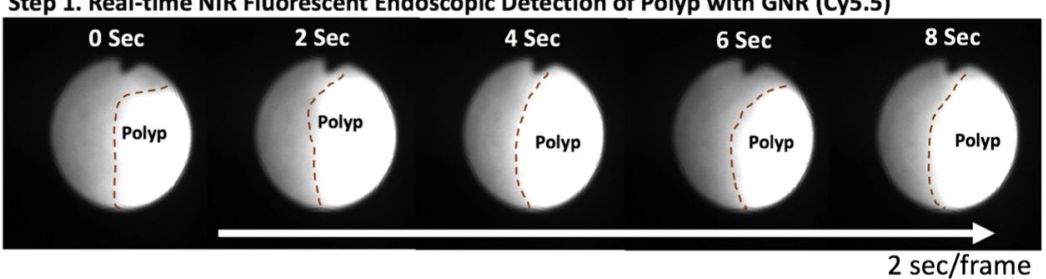

Step 2. Real-time Images of Endoscopic Photothermal Therapy

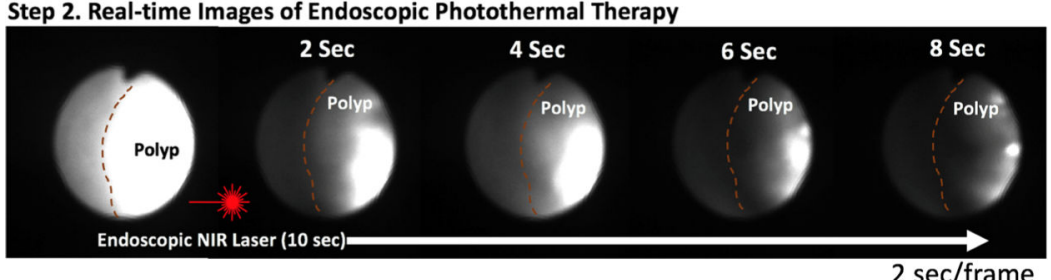

Figure 3.

In vivo real-time endoscopic photothermal ablation of a colon polyp using targeted dualmodal GNR in a TS4 CRE/ APC ${ }^{\text {lox } \triangle 468}$ colon cancer mouse model. Step 1. Real-time detection of accumulated GNRs (Cy5.5) in the colon polyps in the NIRF endoscope. Step 2. Real-time photothermal ablation of the NIRF endoscope-detected polyp with GNRs. 
(a)

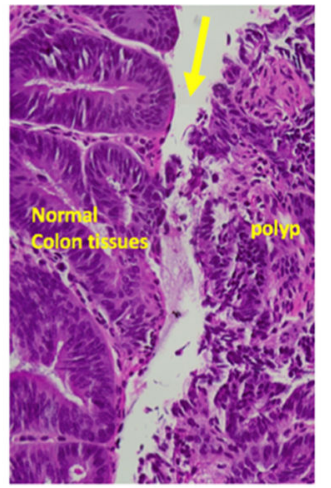

(c)

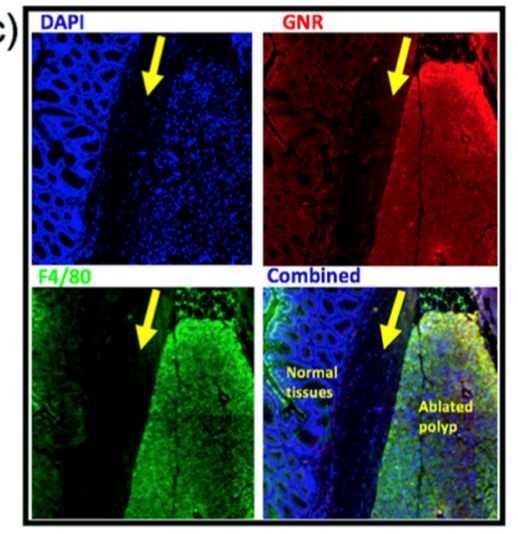

(b)
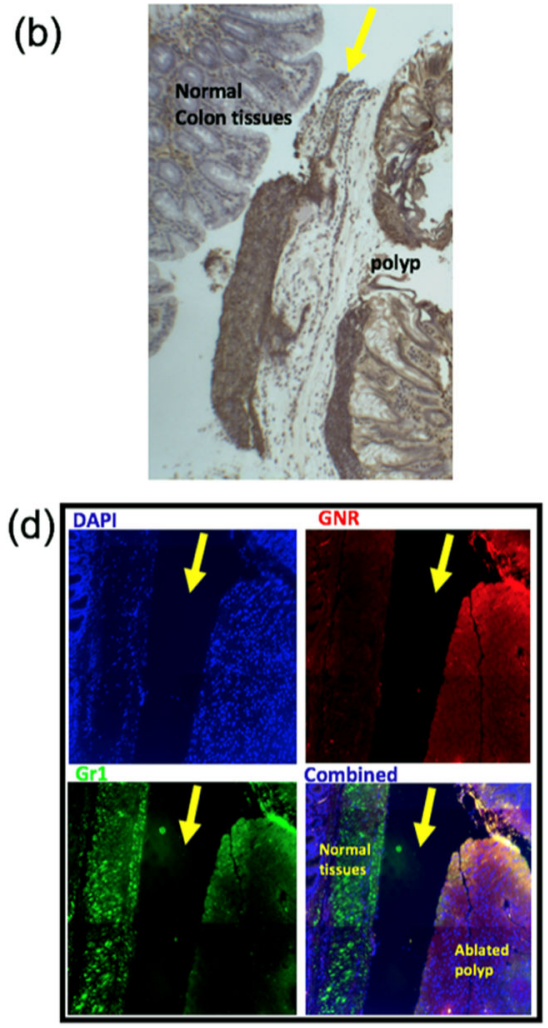

Figure 4.

Histological analysis of tissues treated with NIRF endoscopic image-guided PTA with dualmodal GNRs in the transgenic colorectal cancer mouse model. Representative (a) H\&E-and (b) TUNEL-stained tissue sections of the treated mice. (c,d) Intravital microscopy of small polyps after iv injection of dual-modal GNRs (at $24 \mathrm{~h}$ after injection) and NIRF endoscopic image-guided PTA. Blue (DAPI: extracellular matrix), red (Cy5.5: dual-modal GNRs), and green (F4/80: macrophages or Gr1: neutrophils). Yellow arrows are indicating the interface area between a polyp and normal tissues. 

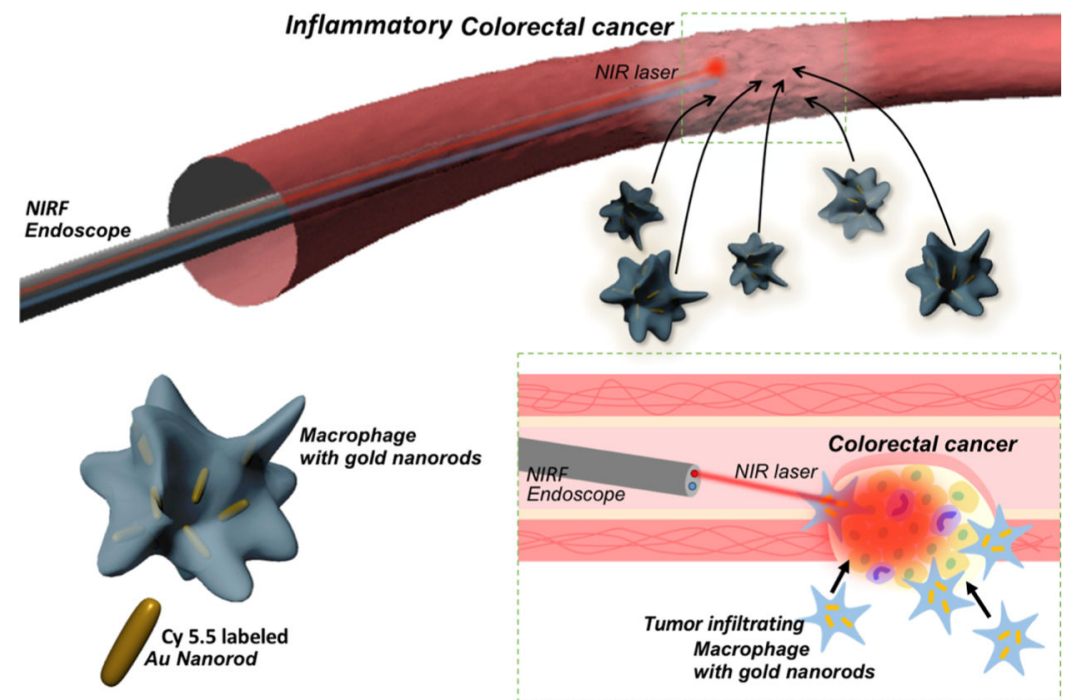

\section{Scheme 1.}

Schematic Illustrations of NIRF Endoscopic Image-Guided PTA Therapy with Dual-Modal GNRs for the Treatment of Colorectal Cancer ${ }^{a}$

${ }^{a} \mathrm{IV}$ injected GNRs are recognized as foreign by macrophages and internalized into macrophages through phagocytosis. The macrophages, including GNRs, are accumulated in tumor sites. The GNRs are imaged through an NIRF endoscope to specifically irradiate only the cancer cells with an NIR laser (i.e., $808 \mathrm{~nm}$ ). The GNRs accumulated in the tumor tissue generate heat to ablate cancer cells upon endoscopic laser irradiation. 